\title{
The orbit of 2010 TK7: possible regions of stability for other Earth Trojan asteroids
}

\author{
R. Dvorak ${ }^{1}$, C. Lhotka ${ }^{2}$, and L. Zhou ${ }^{3}$ \\ 1 Universitätssternwarte Wien, Türkenschanzstr. 17, 1180 Wien, Austria \\ 2 Namur Center for Complex Systems, Départment de Mathématique, 8, Rempart de la Vierge, 5000 Namur, Belgium \\ 3 Department of Astronomy \& Key Laboratory of Modern Astronomy and Astrophysics in Ministry of Education, Nanjing University, \\ Nanjing 210093, PR China \\ e-mail: zhouly@nju.edu.cn
}

Received 1 November 2011 / Accepted 1 February 2012

\begin{abstract}
The first Earth Trojan has been observed and found to be on an interesting orbit close to the Lagrange point L4. In the present study, we therefore perform a detailed investigation of the stability of its orbit and moreover extend the study to give an idea of the probability of finding additional Earth Trojans. Our results are derived using three different approaches. In the first, we derive an analytical mapping in the spatial elliptic restricted three-body problem to find the phase space structure of the dynamical problem. We then explore the stability of the asteroid in the context of the phase space geometry, including the indirect influence of the additional planets of our Solar system. In the second approach, we use precise numerical methods to integrate the orbit forward and backward in time in different dynamical models. On the basis of a set of 400 clone orbits, we derive the probability of capture and escape of the Earth Trojan asteroid 2010 TK7. To this end, in the third approach we perform an extensive numerical investigation of the stability region of the Earth's Lagrangian points. We present a detailed parameter study of possible stable tadpole and horseshoe orbits of additional Earth Trojans, i.e. with respect to the semi-major axes and inclinations of thousands of fictitious Trojans. All three approaches lead to the conclusion that the Earth Trojan asteroid 2010 TK7 finds itself in an unstable region on the edge of a stable zone; additional Earth Trojan asteroids may be found in this regime of stability.
\end{abstract}

Key words. celestial mechanics - chaos - minor planets, asteroids: individual: 2010 TK7 - methods: analytical - methods: numerical

\section{Introduction}

The giant planets Jupiter and Neptune are known to host Trojan asteroids, and Mars (Bowell et al. 1990) has also been found to host several co-orbiting asteroids. These bodies move in the same orbit as the planets, but about $60^{\circ}$ either ahead or behind the planet close to the so-called Lagrange points $L_{4}$ or $L_{5}$ (see Fig. 1). It was a great surprise to discover the first Earth Trojan $2010 \mathrm{TK}_{7}$ (Mainzer et al. 2011). Although many studies had long demonstrated the principal possibility of their existence, until this event all attempts at finding one had been unsuccessful. Another small asteroid in the 1:1 mean motion resonance (MMR) with the Earth had been found earlier in 1986, by Duncan Waldron. However this asteroid (3753 Cruithne) was later identified as a celestial body in a horseshoe orbit around both equilateral Lagrange points of the Earth. It is thus not a "real" Trojan in the sense of its original definition (the same is true for the recently found asteroid $2010 \mathrm{SO}_{16}$ ).

Many theoretical studies have established the stability of the Lagrange points in simplified models, i.e. the studies of Rabe (1967); Bien \& Schubart (1984); Lhotka et al. (2008) and Érdi et al. (2009) as well as many others. Ever since, extensive numerical studies have been undertaken to find the extension of the stability regions around the equilibrium points of the planets, e.g. Schwarz et al. (2004), Dvorak \& Schwarz (2005), Robutel et al. (2005), Freistetter (2006), Dvorak et al. (2007). Particularly important results have been provided by
Mikkola \& Innanen (1992), Tabachnik \& Evans (2000), Brasser \& Letho (2002) and Scholl \& Marzari (2004). In Mikkola \& Innanen (1992) and Zhang \& Innanen (1995) the authors found that Venus, Earth, and Mars can host co-orbital asteroids up to 10 Myr. According to the investigation of Tabachnik \& Evans (2000), Earth's Trojans are on stable orbits when their inclinations are relatively low $\left(i<16^{\circ}\right)$; a second stability window exists according to them for $16^{\circ}<i<24^{\circ}$.

Morais \& Morbidelli (2002) studied the orbital distribution of the near-Earth asteroids (NEAs) that are found for part of their orbit at the 1:1 MMR with the Earth. Schwarz \& Dvorak (2011) investigated the possibility of captures of asteroids by the terrestrial planets into the 1:1 MMR and found many temporary captures including jumping Trojans (Tsiganis et al. 2000). As we show $2010 \mathrm{TK}_{7}$ also has such an interesting captured orbit.

In our investigations here - initiated by the finding of 2010 $\mathrm{TK}_{7}-$ we concentrate on Earth Trojans. We present three different approaches to help us clarify the stability problem of these asteroids. In Sect. 2 we perform analytical studies in a simplified model that is valid on short timescales. We investigate the phase structure and the influence of additional perturbations in the simplified model. In Sect. 3 we study in great detail the actual orbit of $2010 \mathrm{TK}_{7}$, together with 400 clone orbits, to help us assess with greater certitude the probability of both the capture and escape of this Trojan asteroid. To this end, we perform in Sect. 4 an extensive numerical investigation of the parameter space relevant to determining the stable and unstable regions close to the 


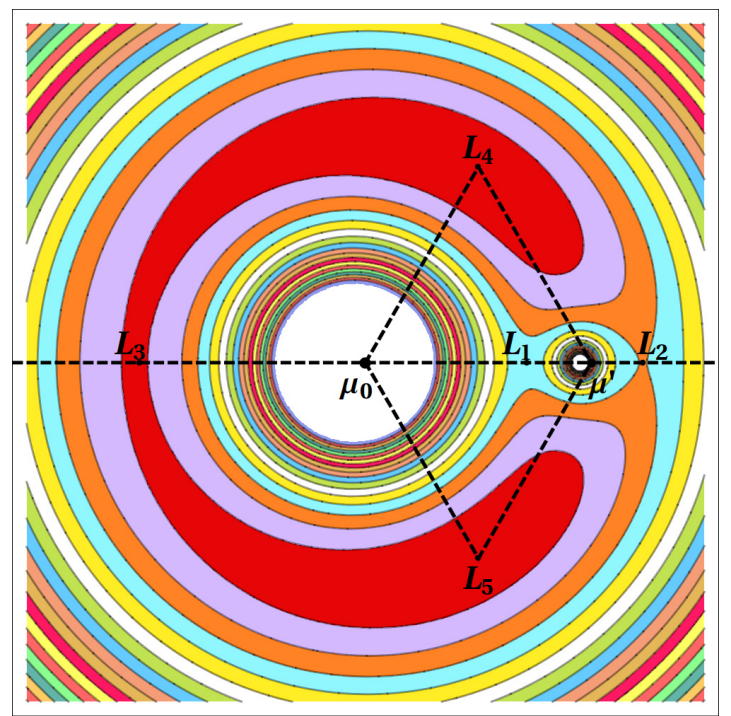

Fig. 1. Geometry of the circular restricted three-body problem in a rotating coordinate system mass of the Sun $\mu_{0}$ and the Earth $\mu^{\prime}$; the unstable equilibria $L_{1,2,3}$ are located on the abscissa; the stable points $L_{4}$ and $L_{5}$ are found $60^{\circ}$ ahead and behind the primary mass $\mu^{\prime}$. The color code defines the areas of the equipotential. In the elliptic problem, the rotating coordinate system has to replaced by a non uniformly rotating and pulsating reference frame.

equilibrium point $L_{4}{ }^{1}$ and show the interplay between secular resonances and the stability of motion. Our summary and conclusions for the present study can be found in Sect. 5 .

\section{A symplectic mapping model}

The Hamiltonian describing the motion of $2010 \mathrm{TK}_{7}$ in the spatial elliptic restricted three-body problem (Sun-Earth-asteroid) takes the form

$$
H=H_{\mathrm{Kep}}+T+\mu^{\prime} R\left(a, e, i, \omega, \Omega, M, M^{\prime} ; p^{\prime}\right),
$$

where $H_{\text {Kep }}$ denotes the Keplerian part, the function $\mu^{\prime} R$ is the perturbation due to the Earth with mass $\mu^{\prime}$, and the variable $T$ is the action conjugated to time (assuming that the mean motion $n^{\prime}$ of the Earth is equal to one). Moreover, the parameters $a, e, i, \omega, \Omega, M$ are the semi-major axis, the eccentricity, the inclination, the perihelion, the longitude of the ascending node, and the mean anomaly of the asteroid, respectively, while $M^{\prime}$ denotes the mean anomaly of the Earth.

In contrast to the classical expansion techniques of the perturbing function $R$, we do not replace the remaining orbital parameters of the Earth $p^{\prime}=\left(a^{\prime}, e^{\prime}, i^{\prime}, \omega^{\prime}, \Omega^{\prime}\right)$ with their numerical values but instead keep them as free parameters in the ongoing calculations. The simple reason for this is we are able to investigate their influence on the dynamics of the massless asteroid with time, i.e. to see their influence on the phase space geometry later on. For the mapping approach, we set the system of constants (gravitational constant $G$ and the total mass of the system) equal to unity such that $G\left(\mu_{0}+\mu^{\prime}\right)=1$, which also implies that $a^{\prime}$ as well as $a$ are close to unity and in these dimensionless units, one revolution period of the Earth takes $P=2 \pi$. Furthermore, we

\footnotetext{
1 Both Lagrange points have in principal the same dynamical behavior, as we know from earlier studies e.g. Nesvorny \& Vokrouhlický (2009), Zhou et al. (2009).
}

use as action-angle variables the modified Delaunay variables, which are defined in terms of the classical Delaunay variables by

$l=M, g=\omega, h=\Omega$

$L=\sqrt{a}, G=L \sqrt{1-e^{2}}, H=G \cos (i)$

as

$\lambda_{1}=l+g+h, \lambda_{2}=-g-h, \lambda_{3}=-h$

$\Lambda_{1}=L, \Lambda_{2}=L-G, \Lambda_{3}=G-H$

(and similar for the primed variables of the Earth). In this setting, the Hamiltonian given in Eq. (1) becomes

$H=-\frac{1}{2 \Lambda_{2}^{2}}+T+\mu^{\prime} R\left(\lambda, \Lambda, \lambda_{1}^{\prime} ; q^{\prime}\right)$,

where we used the short-hand notation $\lambda=\left(\lambda_{1}, \lambda_{2}, \lambda_{3}\right)$ and $\Lambda=\left(\Lambda_{1}, \Lambda_{2}, \Lambda_{3}\right)$. In addition we abbreviate the parameter vector $q^{\prime}=\left(\lambda_{2}^{\prime}, \lambda_{3}^{\prime}, \Lambda_{1}^{\prime}, \Lambda_{2}^{\prime}, \Lambda_{3}^{\prime}\right)$ to define the vector of modified Delaunay variables for the Earth. Next, we implement a symplectic change of coordinates and momenta suitable to describing the motion of the asteroid close to the 1:1 MMR. We define the resonant angle and conjugated momenta as

$\tau=\lambda_{1}-\lambda_{1}^{\prime}, T=\Lambda_{1}$,

while the other variables transform by the identity

$\lambda_{2}=\varphi, \lambda_{3}=\theta, \Lambda_{2}=\Phi, \Lambda_{3}=\Theta$,

and $T^{\prime}=\Lambda_{1}-\Lambda_{1}^{\prime}$. We replace $\lambda_{1}$ in Eq. (2) according to Eq. (3) and implement the standard averaging procedure over the fast angle $\lambda^{\prime}$ via the formula

$\tilde{H}=-\frac{1}{2 T^{2}}+\frac{1}{P} \int_{0}^{P} \mu^{\prime} R\left(\lambda, \Lambda, \lambda_{1}^{\prime} ; q^{\prime}\right) \mathrm{d} \lambda^{\prime}$,

with $P=2 \pi$, to get the averaged Hamiltonian function of the form $\tilde{H}=\tilde{H}\left(\psi, \Psi ; q^{\prime}\right)$ with $\psi=(\tau, \varphi, \theta)$ and $\Psi=(T, \Phi, \Theta)$. Our aim is to construct a symplectic mapping that transforms the state vector $\left(\psi_{k}, \Psi_{k}\right) \equiv\left(\tau_{k}, \varphi_{k}, \theta_{k}, T_{k}, \Phi_{k}, \Theta_{k}\right)$ at discrete times $k$ (multiples of $P$ ) into the state vector $\left(\psi_{k+1}, \Psi_{k+1}\right)$ at times $k+1$. For this reason, we define the generating function

$$
\begin{aligned}
W_{q^{\prime}} & =W_{q^{\prime}}\left(\tau_{k}, \varphi_{k}, \theta_{k}, T_{k+1}, \Phi_{k+1}, \Theta_{k+1} ; q^{\prime}\right) \\
& =\psi_{k} \cdot J_{k+1}+2 \pi \tilde{H}\left(\psi_{k}, J_{k+1} ; q^{\prime}\right),
\end{aligned}
$$

where the symbol · is the dot product. As shown and used in Hadjidemetriou (1992), Hadjidemetriou (1999), Hadjidemetriou \& Voyatzis (2000) and Ferraz-Mello (1997) the generating function defines a mapping of the form

$$
\begin{aligned}
\tau_{k+1} & =\frac{\partial W_{q^{\prime}}}{\partial T_{k+1}}, \varphi_{k+1}=\frac{\partial W_{q^{\prime}}}{\partial \Phi_{k+1}}, \theta_{k+1}=\frac{\partial W_{q^{\prime}}}{\partial \Theta_{k+1}}, \\
T_{k} & =\frac{\partial W_{q^{\prime}}}{\partial \tau_{k}}, \Phi_{k}=\frac{\partial W_{q^{\prime}}}{\partial \varphi_{k}}, \Theta_{k}=\frac{\partial W_{q^{\prime}}}{\partial \theta_{k}} .
\end{aligned}
$$

The system of Eq. (5) defines a symplectic change of coordinates on the Poincaré surface of section obtained by the averaged system defined by Eq. (4). The set of variables $(\Psi, \psi)$ is related to the mean orbital elements of the asteroid $2010 \mathrm{TK}_{7}$ via another generating function (not derived here), which defines the averaging process given in Eq. (4). The system of Eq. (5) therefore describes the evolution of the mean orbital elements of the asteroid at discrete times $t=k \cdot P$. We note that the system is implicit to preserving the Hamiltonian structure of the original 


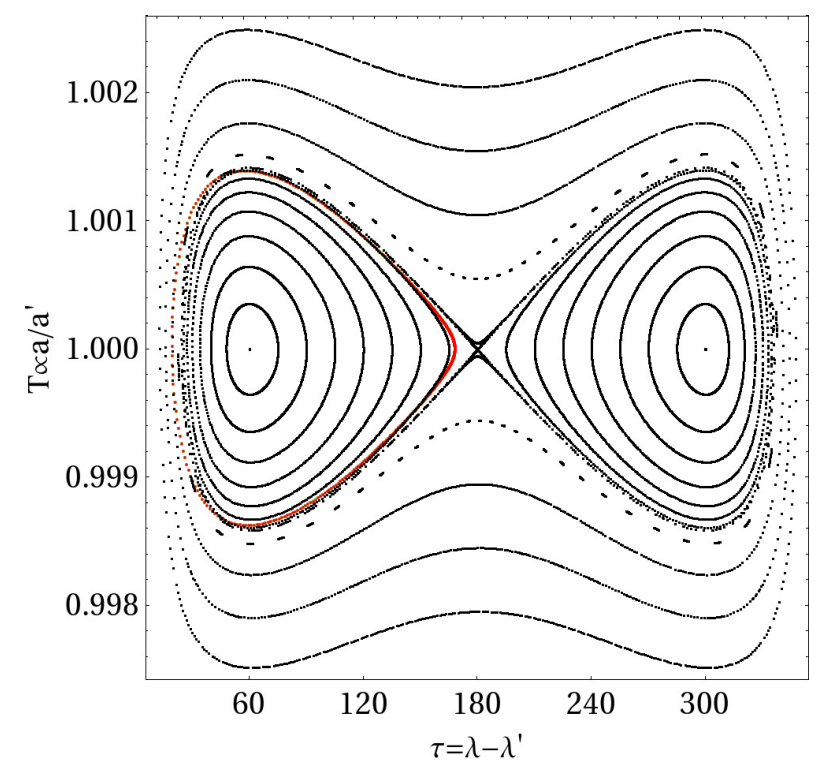

Fig. 2. Phase portrait, projected to the $(\tau, T)$-plane. The Earth is located at $(0,1)$, the fixed points $L_{4}, L_{3}$ and $L_{5}$ (corresponding to the equilibria of the averaged system) are situated $\left(60^{\circ}, 1\right),\left(180^{\circ}, 1\right)$, and $\left(300^{\circ}, 1\right)$, respectively. The projection of the mean orbit of the asteroid $2010 \mathrm{TK}_{7}$ for \pm 6000 years is shown in red.

problem. For given initial conditions $\left(\psi_{k=0}, \Psi_{k=0}\right)$, it can either be iterated by solving the system of difference equations implicitly for $\left(\Psi_{k+1}, \psi_{k+1}\right)$ or by using more sophisticated procedures as described e.g. in Lhotka (2009).

A typical projection of the phase portrait onto the $(\tau, T)$-plane is shown in Fig. 2. The lines in black were obtained by varying $\tau$ within the range $\left(0^{\circ}, 360^{\circ}\right)$ along $T=1$. The plot also shows the mean orbit of $2010 \mathrm{TK}_{7}$ in red, which should be compared with Fig. 2 of Connors et al. (2011): while these authors derive the red curve by numerical averaging, the averaged orbit for our present approach is based on Eq. (5).

A well-established result is that, e.g. Morais \& Morbidelli (2002); Brasser \& Letho (2002); Scholl et al. (2005), the influence of the other Solar system bodies, i.e. the direct influence of the major planets, affects the motion of the asteroids on long timescales. The mapping does not take into account these direct effects. Thus, the mean orbit as shown in Fig. 2 can only been seen as a first approximate solution of the problem. Moreover, the simulations were performed using low order expansions of the perturbing function, since we wished to maintain the dependence of the model on the orbital parameters of the Earth. The resulting mapping model is therefore only valid within a good convergence regime of the Fourier-Taylor series expansions used to approximate $R$ in Eq. (1). In addition, since the orbital parameters of the asteroid $2010 \mathrm{TK}_{7}$ may reach high values (as found from numerical simulations), the error in the approximation of the series expansions may exceed the cumulative effect of the perturbation effects due to the Solar system and may also change the picture dramatically on longer time scales. We are performing an ongoing investigation to estimate of the error terms and higher order series expansions, together with their influence on the long-term dynamics of the asteroid $2010 \mathrm{TK}_{7}$.

\section{The orbit of $2010 \mathrm{TK}_{7}$}

The Earth Trojan $2010 \mathrm{TK}_{7}$ is fundamentally a NEA, and its orbital elements (listed in Table 1) can also be found on the
Table 1. The Keplerian elements of $2010 \mathrm{TK}_{7}$ given at epoch JD 2455800.5 .

\begin{tabular}{lccc}
\hline \hline & Value & $1-\sigma$ variation & Unit \\
\hline$a$ & 1.00037 & $2.546 \times 10^{-7}$ & $\mathrm{AU}$ \\
$e$ & 0.190818 & $9.057 \times 10^{-7}$ & \\
$i$ & 20.88 & $7.274 \times 10^{-5}$ & $\mathrm{deg}$ \\
$\Omega$ & 96.539 & $1.842 \times 10^{-4}$ & $\mathrm{deg}$ \\
$\omega$ & 45.846 & $2.309 \times 10^{-4}$ & $\mathrm{deg}$ \\
$M$ & 217.329 & $1.848 \times 10^{-4}$ & $\mathrm{deg}$ \\
\hline
\end{tabular}

Notes. The elements and 1- $\sigma$ variations are taken from the AstDyS (see text). The covariance matrix is also taken from the same website.
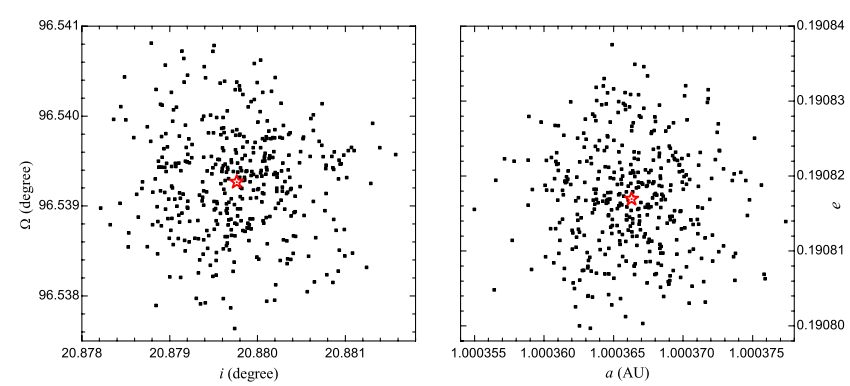

Fig. 3. The initial conditions of clone orbits. Dots are for 400 clones, and the nominal orbit is indicated by the red star. In two panels, we show here only the inclination, ascending node, semimajor axis, and eccentricity.

AstDyS (Asteroids - Dynamic Site) website ${ }^{2}$. At first glance, this object has a large eccentricity $(\sim 0.19)$, and it is reasonable to suspect that it is on an unstable orbit. To check the orbital stability of this object, we perform numerical simulations of the orbit.

We adopt two dynamical models in our simulations. Both models contain the Sun and eight planets from Mercury to Neptune. They differ from each other in terms of the different settings of the Earth-Moon system. In one model, the EarthMoon system is simply treated as one mass point with the combined mass of the Earth and the Moon at the barycenter of the system. In another model, the Earth and the Moon are regarded as two separate objects as in reality. Hereafter, we denote the former as the EMB model and the latter as the E+M model for short. By comparing these two models, we may testify the reliability of the model that we adopt in this paper and adopted by Connors et al. (2011) in their numerical simulations.

Considering the uncertainties in the observations and in the orbital determination, it is necessary to study clone orbits within the error bars. In addition to the nominal orbit, 400 clone orbits are generated using the covariance matrix given by the AstDyS website. As listed in Table 1, the errors are very small, therefore the initial conditions of the clone orbits are very close to the nominal orbit as shown in Fig. 3. We present in Fig. 3 the distribution of $a, e, i, \Omega$, and note that the other two elements $M$ and $\omega$ that are not illustrated here have similar distributions according to the corresponding uncertainties listed in Table 1.

Starting from the initial conditions generated in the above way, we integrate their orbits up to 1 million years (Myr) in both directions (forward to the future and backward to the past). We used the integrator package Mercury6 (Chambers 1999) and verified some results using the Lie-integrator (Hanslmeier \& Dvorak 1984). The comparison between the results from

${ }^{2}$ http://hamilton.dm.unipi.it 


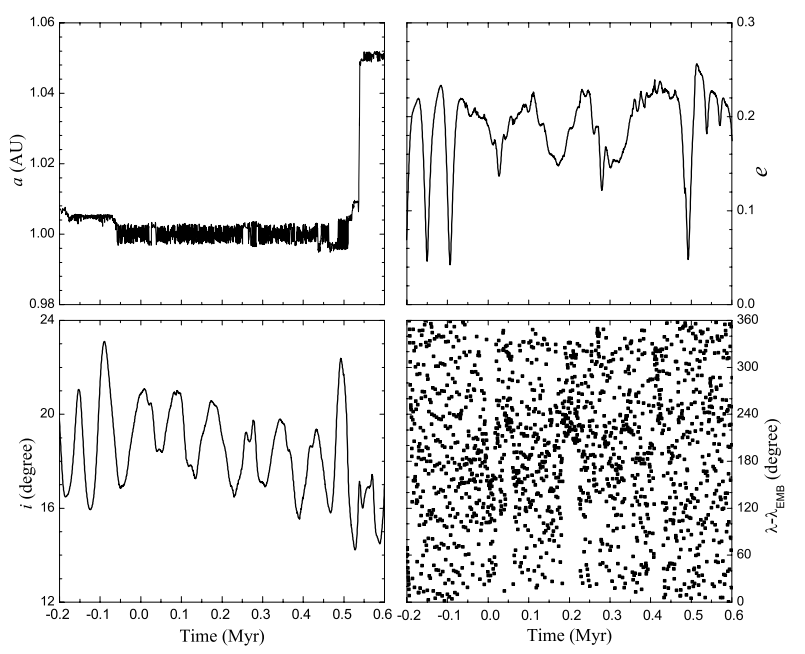

Fig. 4. The temporal evolution of the nominal orbit in 1 million years, both forward and backward. In four panels, we show the evolutions of the semimajor axis (upper left), eccentricity (upper right), inclination (lower left), and the resonant angle (lower right).

different integrators shows that the results are consistent with each other.

For an asteroid on a Trojan-like orbit, the critical angle (resonant angle) is the difference between the mean longitudes of the asteroid $\lambda$ and the corresponding host planet (which here is the Earth-Moon system) $\lambda^{\prime}$, like the $\tau$ in Eq. (3). In the EMB model, the resonant angle is $\lambda-\lambda_{\mathrm{EMB}}$, while in the $\mathrm{E}+\mathrm{M}$ model we calculate the position and velocity of the barycenter of the Earth-Moon system from the orbital elements of the Earth and the Moon, and then compute the resonant angle in the same way as in the EMB model. When the resonant angle $\lambda-\lambda^{\prime}=\lambda-\lambda_{\mathrm{EMB}}$ librates around $60^{\circ}$, i.e. $0^{\circ}<\lambda-\lambda^{\prime}<180^{\circ}$ the asteroid is said to be an $L_{4}$ Trojan, when it librates around $-60^{\circ}$ (or equivalently $300^{\circ}$ ), i.e. $180^{\circ}<\lambda-\lambda^{\prime}<360^{\circ}$ it is an $L_{5}$ Trojan, when the asteroid librates with an amplitude larger than $180^{\circ}$ it is on a horseshoe orbit, and finally when $\lambda-\lambda^{\prime}$ circulates, the asteroid leaves the Trojan-like orbit.

In Fig. 4, we illustrate the temporal evolution of the nominal orbit in the $\mathrm{E}+\mathrm{M}$ model. The first impression obtained from its behavior may be that it is a temporal Earth Trojan, i.e. judging from the resonant angle it was not an Earth Trojan 0.055 Myr before and will not remain on a Trojan-like orbit after 0.37 Myr. It is an $L_{4}$ Trojan for a time interval that is even much shorter, less than 2000 years in the past and less than 17000 years in the future (as partly shown in Fig. 5). While it is a Trojan, the semimajor axis $a$ undergoes regular variations librating around 1.0 AU with a small amplitude, although the variations of both eccentricity $e$ and inclination $i$ reveal the chaotic character of the motion, which is illustrated in Fig. 4.

This chaotic behavior is indicative of not only its sensitivity to the initial conditions but also the dynamical model. To compare the motions predicted by the two models, i.e. the EMB model and E+M model, we show as an example in Fig. 5 the evolution of the resonant angle of the nominal orbit for both models. Around the starting point, two curves representing the motions in two models are almost the same, so that they overlap each other exactly. However, the difference between them becomes distinguishable only after about 2000 years in both directions. This difference on one hand arises from the different settings of the models, and on the other hand, from the chaotic character of the motion. In this sense, it is impossible to draw

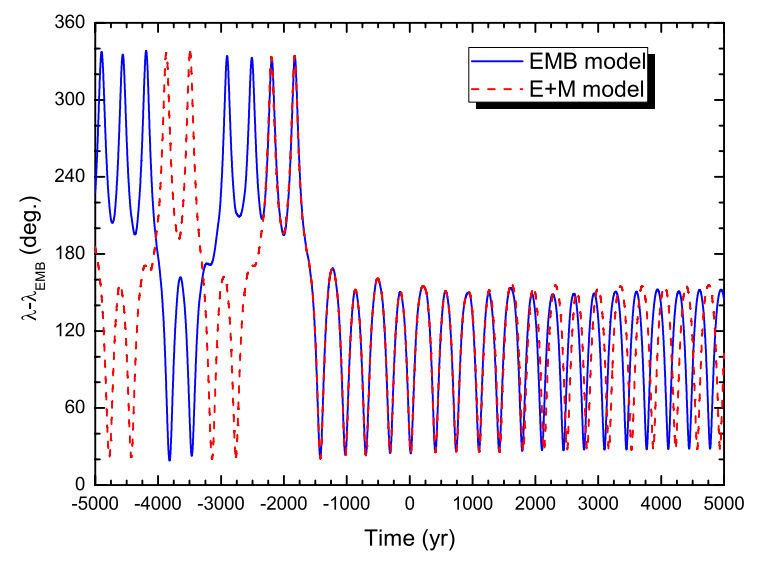

Fig. 5. The resonant angle of the nominal orbit in two models. The difference between them is readily apparent very early in the simulating process ( $\sim 2000$ years) mainly due to the chaotic character of the motion (see discussion in text).

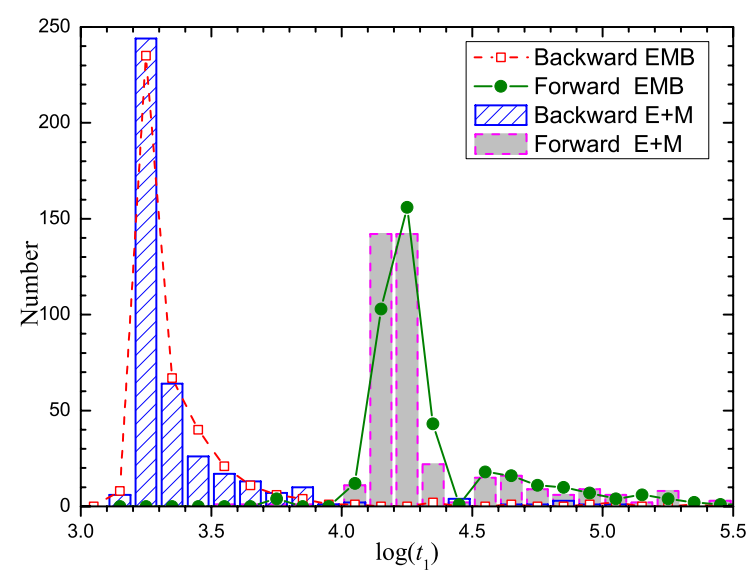

Fig. 6. The distribution of the time when clone orbits leave from the current $L_{4}$ region. The escape time from the tadpole region is given in years on a logarithm scale.

any convincible conclusion about these two dynamical models on long time scales by comparing merely the motions of an individual orbit in the two models. Hence we now turn to analyze statistically the four hundred clone orbits.

As we mentioned above, 400 clone orbits are calculated in both models and in both directions of time. At the starting moment $t=0$, these clones are around the $L_{4}$ Lagrange point as the asteroid $2010 \mathrm{TK}_{7}$, but during the course of their evolutions, the objects may jump from the $L_{4}$ region to the $L_{5}$ region, or move from tadpole to horseshoe orbit, and even escape from the Trojan-like orbit (the 1:1 MMR). To examine the motion, we check the resonant angle $\lambda-\lambda_{\text {EMB }}$ at each step during our simulations. When for the first time $\lambda-\lambda_{\mathrm{EMB}}$ is larger than $180^{\circ}$, the object is assumed to have left the $L_{4}$ region, and we denote the time at that moment as $t_{1}$. When $\lambda-\lambda_{\mathrm{EMB}}$ for the first time reaches $360^{\circ}$, the object escapes the 1:1 MMR, and we denote this moment as $t_{2}$.

The $t_{1}$ and $t_{2}$ of 400 clone orbits are summarized in Figs. 6 and 7 . For $t_{1}$, most of the clones will leave the $L_{4}$ region about $1.5 \times 10^{4}$ years in the future, while the backward integrations indicate that the clones "entered" the $L_{4}$ region about $1.8 \times$ $10^{3}$ years ago (Fig. 6).

In the backward integrations, the earliest escape from the $L_{4}$ region happens at $t \sim 1440$ years in both the $\mathrm{E}+\mathrm{M}$ and 


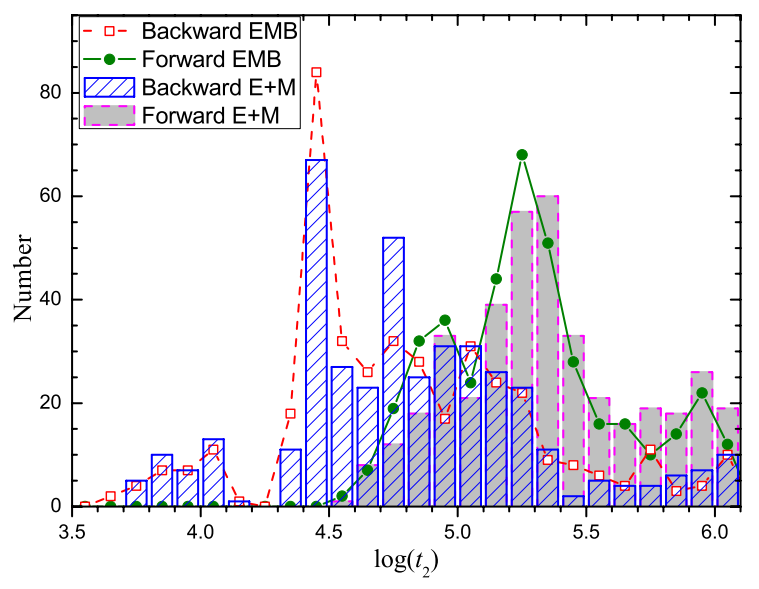

Fig. 7. The distribution of the time when clone orbits escape from the 1:1 MMR, both in forward and backward integrations. The escape time is given in years on a logarithmic scale.

EMB models. However there are only a few such orbits. Most of them escape in the time range between $t \sim 1680$ years and $t \sim 1820$ years, which causes the peak centered on $\log \left(t_{1}\right)=3.25$ in Fig. 6. From Fig. 5, we may derive the libration period of the resonant angle to be around 350 years $^{3}$, and we also note that the shift in the orbit from the $L_{4}$ to the $L_{5}$ region happens when the resonant angle reaches a maximum in a libration period. The libration period and amplitude must be tuned by other periodic effects (e.g. secular resonances). After a number of complete periods of evolution, the resonant angles of some clone orbits reach their maxima, and they do not librate back but escape from there towards the neighborhood of $L_{5}$. After another complete libration period, many more clone orbits escape in the same way as before. This is why the escapes from the $L_{4}$ region seem to happen more or less suddenly (at $t \sim 1440+350=1790 \approx 10^{3.25}$ years). All clone orbits are in a tiny region confined by the error bars, so that they suffer nearly the same dynamical effects in a short timespan. For the clones that remain in the region longer, the phase space occupied by them increases in size, the clones thus suffer different dynamical effects, and the clone escape times consequently diverge.

The distributions of $t_{1}$ for the EMB model and the E+M model in Fig. 6 match each other very well, hence we may draw another conclusion here that the difference between these two dynamical models is negligible, or in other words, the simplified EMB model is a reasonable and reliable model for investigating the Earth Trojans' long-term dynamics. In particular, we note that the asteroid $2010 \mathrm{TK}_{7}$ and the clones all have very large libration amplitudes around the $L_{4}$ and/or $L_{5}$ point (see for example Fig. 5), i.e. they may approach the Earth-Moon system (the mean longitude difference between them may be less than $20^{\circ}$ ). However, even when an Earth Trojan approaches the Earth and comes within $10^{\circ}$ away from the Earth in mean longitude, the distance between the asteroid and the Earth is about $0.18 \mathrm{AU}$, which is still about 70 times larger than the distance between the Earth and the Moon. Taking into account the inclination of the Earth Trojan's orbit, the distance between the Trojan and the Earth is even larger.

\footnotetext{
${ }^{3}$ Owning to the large libration amplitude, this value is nearly 50 percent longer than the synodic period of a tadpole orbit, which can be estimated using the formula $(27 \mu / 4)^{-1 / 2}$ (Murray \& Dermott 1999). For an Earth Trojan, the mass ratio $\mu=3.04 \times 10^{-6}$ and the period is $\sim 220$ years.
}

As for the $t_{2}$ distribution in Fig. 7, we see that the peak of the escape time in the backward integrations occurs at $\sim 3.0 \times$ $10^{4}$ years. As we mentioned above, the Earth Trojan $2010 \mathrm{TK}_{7}$ should be a temporal Trojan. The capture of this asteroid onto the 1:1 MMR with the Earth most probably happened 30000 years ago. In the forward integrations, most of clones will escape from the MMR after around $2.0 \times 10^{5}$ years. The total lifetime of this asteroid in the 1:1 MMR is shorter than 0.25 Myr. Again, the two models $\mathrm{E}+\mathrm{M}$ and $\mathrm{EMB}$ produce nearly the same distributions.

In our calculations, the time interval during which the clones are $L_{4}$ Earth Trojans is typically a little shorter than $\sim 17000$ years. As in the case of the lifetime of clones staying in the 1:1 MMR, there are 10 clones surviving $1 \mathrm{Myr}$ in the backward integrations in both models, while for the forward integrations, 12 in the EMB model and 19 in the E+M model stay in the MMR until the end of integrations. However, none of the clones survive in both temporal directions. Simply put fewer than 5 percent of the clones remain in the MMR up to $1 \mathrm{Myr}$. Morais \& Morbidelli (2002) calculated the probability of a NEA being captured into the 1:1 MMR ("coorbital orbit" in their paper), and they found that each episode of a NEA being coorbital is on average 25000 years and none lasts longer than 1 Myr. Our result does not conflict with their conclusion, because our calculations are for the individual asteroid $2010 \mathrm{TK}_{7}$, and its eccentricity $(\sim 0.2)$ is smaller than the typical eccentricity in their samples (most of them have $e>0.28$ ).

Some fluctuations in the distribution of $t_{1}$ and $t_{2}$ (by "fluctuation" we mean more peaks that deviate from a normal distribution) can be found in both models in Figs. 6 and 7. In these figures, the time is given on a logarithmic scale to include a wider time interval. If we plot the time linearly, these fluctuations have a periodic character, implying that some periodic mechanisms (e.g. secular resonances) affect the libration amplitudes. However, the creation of a secular resonance map is complicated and a task that leave to a future paper, although we show our first results in Sect. 4.3.

\section{Determination of the stable regions}

As pointed out in the previous sections, the orbit of $2010 \mathrm{TK}_{7}$ is not very stable. We now investigate how to determine the extent of the stable regions around the stable equilibrium points. This can be done in a realistic dynamical model only with the aid of numerical integrations where the stability of the orbits of many fictitious Trojans is checked. The dynamical model used was a "truncated" planetary system with the planets Venus to Saturn (Ve2Sa). The simple reason for not taking into account all the planets is that this would have required at least four times longer CPU times on our computers. It is mainly due to the orbit of the innermost planet Mercury which demands a step size of one quarter of that of Venus ${ }^{4}$. Test computations of the selected stable and unstable orbits showed that the qualitative behavour of these orbits is not a different one, and consequently also the extent of the stable region can be regarded as the "real" one. It means that the gravitational perturbation of Mercury on a Trojan for this study is negligible. The Moon was not explicitly included in the integration but the barycenter of the Earth-Moon system and their respective masses were taken as one body. As we have shown in Chap. 3, there are only very small differences on long timescales when one integrates the Trojan orbits in these models. In contrast Jupiter's direct perturbation on an Earth Trojan is

${ }_{4}$ The inclusion of Uranus and Neptune would not dramatically change this integration time. 


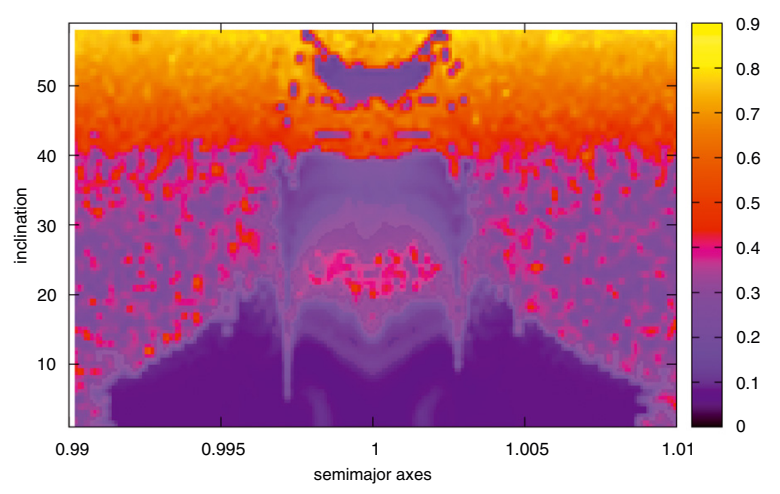

Fig. 8. Stability diagram for Earth Trojans with $i<60$ (y-axes) for different initial semimajor axes ( $x$-axes) of the fictitious Trojans; the color stands for the maximum eccentricity during the integration of $10^{6}$ years.

very large and cannot be neglected especially for large librations that bring the Trojan close to the Lagrange point $L_{3}$.

The integration method was the Lie-series method that we used quite often in previous similar investigations; e.g. for Neptune (Zhou et al. 2009, 2011) and also for Uranus' Trojans (Dvorak et al. 2010). This method is based on the studies of Hanslmeier \& Dvorak (1984); Delva (1984), and Lichtenegger (1984) and was slightly modified for our problem (see also Eggl $\&$ Dvorak 2010). It has an automatic step-size control and turned out to be fast and precise.

The initial conditions were chosen in the following way: for the fictitious Trojans, the orbital elements $M, \Omega$, and $e$ were set to the ones of the Earth. The perihelion was always $\omega_{\text {Earth }}+60^{\circ 5}$, the semimajor axes for the Trojan was set to slightly smaller and larger values to cover the stable region (along the connecting line between Sun and the Lagrange point). We started with integrations in the plane of the orbit of the Earth and in additional runs changed the inclinations up to $i=60^{\circ}$.

To distinguish between stable and unstable orbits, we used different indicators: the most straightforward method was to check the eccentricity of the object because there was a sharp cut for $e>0.3$ : any orbit that reached this value during the integration left the area around the Lagrange point (checked by its distance to this point). In another test, we directly computed the escape times and finally we also computed the libration width of the Trojan, which is a well established check of the stability of a Trojan. The length of integration time was thoroughly chosen; although we had an available computer grid the main computations covered only $10^{7}$ years but some tests were undertaken up to $10^{8}$ years.

\subsection{The stable regions for different timescales}

The stability diagram for a cut through the Lagrange point $L_{4}$ is shown in Fig. 8, where we have plotted the maximum eccentricity of the orbits of 100 massless fictitious Trojans for different values of the semimajor axes ( $x$-axes) versus the inclinations $\left(0^{\circ} \leq i \leq 60^{\circ}\right)$ of the Trojan ( $y$-axes). One can see that for small inclinations, the stable region extends along the semimajor axes between $0.991 \leq a \leq 1.009$, then there is a decrease of the size in the region visible up to about $i=20^{\circ}$. Inside this region two finger-like structures - slightly less stable regions - are visible on both sides of the $\mathrm{V}$-shaped structure centered at $a=1$ for $15^{\circ} \leq i \leq 20^{\circ}$. An unstable strip then extends to about $i=26^{\circ}$

\footnotetext{
5 Which means that the position was the Lagrange point $L_{4}$.
}

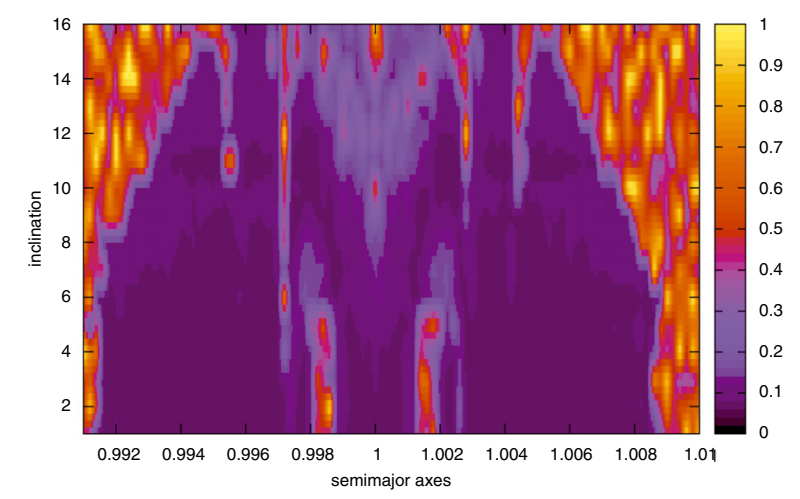

Fig. 9. Caption as for Fig. 8.

with some connection to a larger rectangular stable region with $0.997 \leq a \leq 1.003$ and $26^{\circ} \leq i \leq 40^{\circ}$. Stable symmetric fingers inside this region are clearly visible and build a continuation of the unstable fingers around $a \simeq 0.997$ and $a \sim 1.003$ for the large stable area for $i<20^{\circ}$. For larger inclinations on, $i>40^{\circ}$, the unstable regions extends for all values of the semimajor axes with the exception of a small $U$-shaped stable window around $i=50^{\circ}$. We note that inside this unstable region (red to yellow) sometimes small stable islands seem to appear on both sides of the stable region $i<40^{\circ}$ which - after a longer integration disappear.

For the aforementioned structures visible in the figure we note that similar ones were discovered for the Trojan regions of the outer planets in the papers of Michtchenko \& Ferraz-Mello (2002) and Nesvorný \& Dones (2002) for larger eccentricities. In our paper we investigated the stability for larger inclinations; the different features inside stable regions for Earth Trojans are comparable to the ones found by Zhou et al. (2009) for the Neptune Trojan region. The instabilities inside the stable region are caused by secular resonances - as shown in detail for the Jupiter Trojans by Robutel \& Gabern (2006). In our article we only wish to emphasize - for the moment - the complicated structures which are present. We are performing a detailed analysis of the resonances similar to that of Zhou et al. (2009) or even the aforementioned article of Robutel \& Gabern (2006) but this is taking much more time.

We then extended the integration to $10^{7}$ years in the same dynamical model Ve2Sa. The region of small inclinations is still stable with the same extension along the semimajor axes (Fig. 9). New features, namely unstable vertical strips, appear for different values of $a$ and increasing values of the inclination. For $a \simeq 0.997$ and $a \simeq 1.003$, these regions were already visible as being less stable in the former plot (Fig. 8) for small inclinations. New unstable vertical fingers also appear also for $a \sim 0.995$ and $a \sim 1.005$ and $10^{\circ}<i<16^{\circ}$. Another new characteristic is the appearance of unstable fingers for $a \sim 0.998$ and $a \sim 1.002$ with $i<6^{\circ}$. The V-shaped unstable region centered at $a=1$ was already visible in Fig. 8, but its detailed structures could only be established using a longer integration time.

In Fig. 10 the stable window for $28^{\circ}<i<40^{\circ}$ was studied separately (also for $10^{7}$ years). One can again see that the most stable regions visible through the dark blue - indicating small e-max values - are close to the edges of $a$. These edges are quite irregular and some smaller unstable islands appear inside; the whole stable region is somewhat tattered. We do not show the disappearance of the U-shaped island for $i \sim 50^{\circ}$ for a longer integration time. 


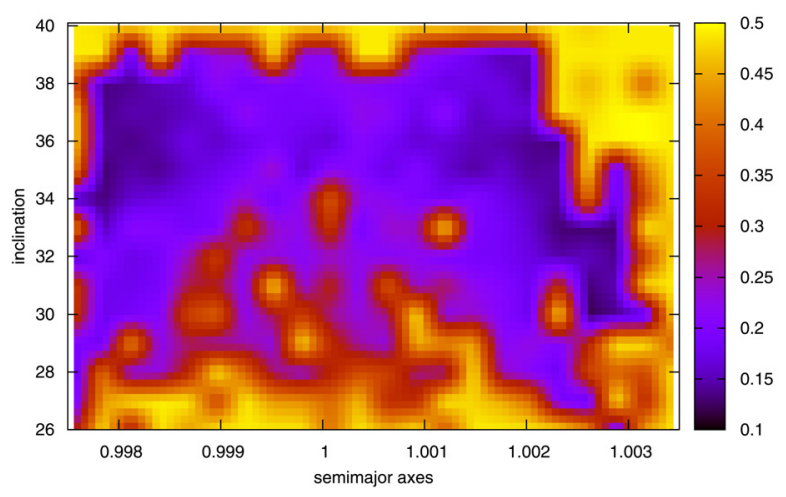

Fig. 10. Stability diagram for the window between $26<i<40$ ( $y$-axes) for different initial semimajor axes ( $x$-axes) of the fictitious Trojan; the color stands for the maximum eccentricity during the integration of $10^{7}$ years.

We note that we disagree, in some aspects, with the results given by Tabachnik \& Evans (2000) who claim that stable regions for Earth Trojans are possible for $24^{\circ}<i<34^{\circ}$; instead we found the stable window is shifted outwards to larger inclinations. We also disagree with Brasser \& Letho (2002) who state that orbits with an inclination of $12^{\circ}<i<25^{\circ}$ are unstable. In the last work, the authors quite nicely determine the secular frequencies involved that lead to unstable motion. We already started additional computations where the determination of the resonances is undertaken, but the detailed analysis of these data will be presented in a future article.

\subsection{The libration amplitudes}

To determine the libration width of the region, we chose for the semimajor axes a grid of $0.995 \leq a \leq 1.005$ for 50 different fictitious bodies equally distributed in the aforementioned interval where the inclination was set to values $0^{\circ} \leq i \leq 56^{\circ}$. The integration time was only $10^{6}$ years for this study. The colors (from blue to yellow) indicate the amplitude of libration; we can see a well defined stable region in the range $0.997 \leq a \leq 1.003$ for inclinations $i \leq 19^{\circ}$. This rectangular region seems to contradict the results shown in Fig. 8, but a closer look shows - through the color of dark yellow - that the libration angle is in the order of $170^{\circ}$; thus, on the edge we have horseshoe orbits that enclose both equilateral Lagrange points. An unstable strip is apparent for $20^{\circ} \leq i \leq 24^{\circ}$ where $2010 \mathrm{TK}_{7}$ is located close to $a \sim 1.0005$ and $i \sim 21^{\circ}$. We then see again a large almost rectangle stable region for $25^{\circ} \leq i \leq 39^{\circ}$ in the same range of the semimajor axes $a$ as the first stable region of tadpole orbits for only slightly inclined Trojan orbits. We note that the edges are sharp and no horseshoe orbits are located there. From $i=40^{\circ}$, no more stable orbits exist with the exception of a small stable window with tadpole orbits for $i \sim 50^{\circ}$; these orbits will be unstable for longer integration times as indicated above.

To illustrate the diversity of orbits we depict three different ones for an inclination $i=1^{\circ}$ of the Trojans' orbits: a tadpole orbit deep inside the stable region (Fig. 12 upper graphs), a horseshoe orbit close to the border of stability (Fig. 12 middle graphs), and an escaping orbit outside the stable region after initially being in a horseshoe orbit (Fig. 12 lower graphs). In the left panels of this figure, the semimajor axes display a periodic change between a maximum and a minimum value close to the semimajor axis of the Earth for the tadpole and the horseshoe orbit. In the middle graph we present the typical behaviour of a body

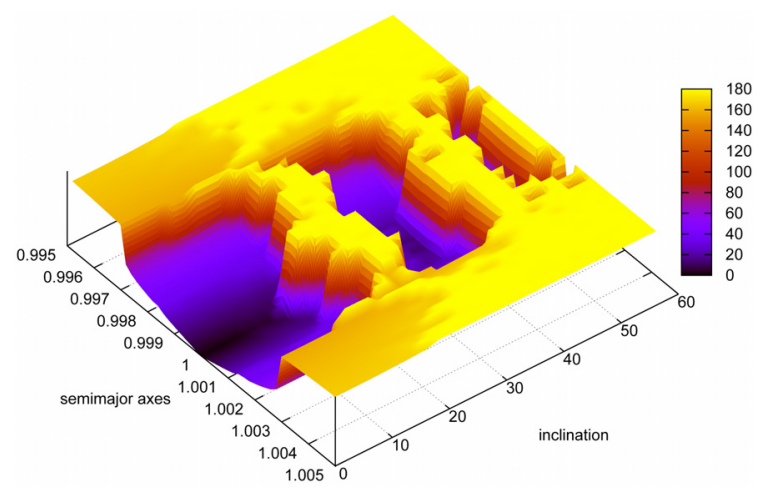

Fig. 11. Libration amplitudes - defined as half of the total libration angle - in the region close to $L_{4}$; the color indicates the largeness of the libration.
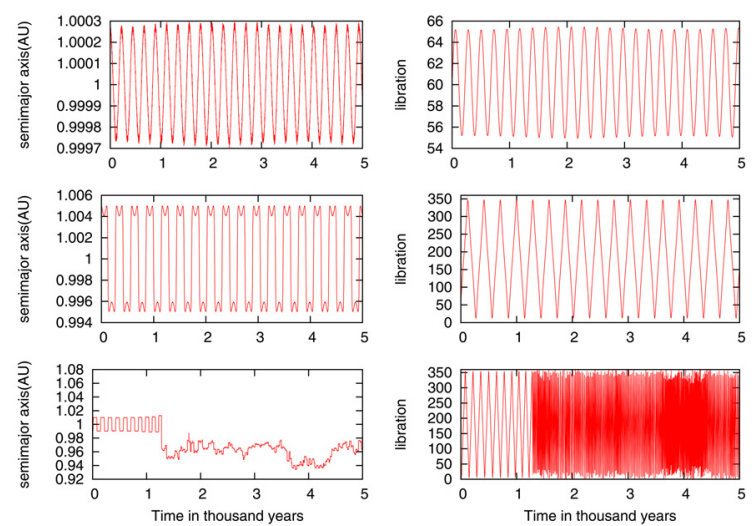

Fig. 12. The temporal evolution of three orbits: a tadpole (upper graphs), a horseshoe, (middle graphs) and an escaping orbit (lower graphs). In six panels we show the evolutions of the semimajor axes (left graphs) and the libration angles (right graphs) over 5000 years.

in a horseshoe orbit in a rotating coordinate system (cf. Fig. 1). Close to the turning point of the orbit, the Trojan finds itself in the vicinity of the Earth, its semimajor axis is larger than that of the Earth, and then - when the libation angle changes from about $10^{\circ}$ to $350^{\circ}$ it reaches values smaller than $a_{\text {Earth }}$; the Trojan is now on the other side of the Earth. In Fig. 12 we show in the lowest panels an unstable orbit that - after leaving the horseshoe orbit - is chaotic with librations between $0^{\circ}$ to $360^{\circ}$.

\subsection{Secular resonances}

We now present our preliminary results about the secular resonances to understand the structure of the stability diagram (Fig. 8). A secular resonance occurs when the precession rate of an object ( $\dot{\varpi}$ or $\dot{\Omega}$ ) equals one of the eigenfrequencies of the system (Murray \& Dermott 1999). In a secular resonance, either the eccentricity or the inclination displays long-term oscillations. The eigenfrequencies of the system can be calculated by means of a linear analysis (Bretagnon 1974) or numerically determined (Laskar 1990).

The reality is more complicated than the model adapted in the linear analysis. The secular perturbations among the planets modify the precession rates. Owning to their high masses, the precession rates of the big planets are almost constant, whereas the precession rates of the inner planets are strongly influenced, in particular by Jupiter and Saturn. In Fig. 13, we show the differences between Jupiter's perihelion longitude $\left(\varpi_{5}\right.$, 


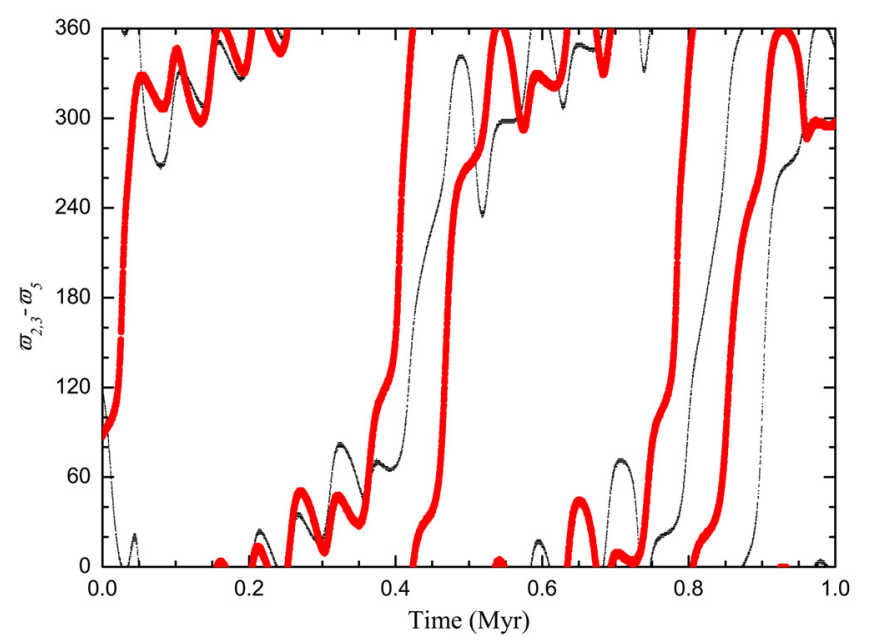

Fig. 13. The differences between the perihelion longitudes of the inner planets and Jupiter. The abscissa is time and the ordinate is $\varpi_{k}-\varpi_{5}$, with $k=2$ for Venus (thin black curve) and $k=3$ for the Earth (thick red curve).

as usually denoted) and the those of Venus $\left(\varpi_{2}\right)$ and the Earth $\left(\varpi_{3}\right)$ in our simulations. During the periods $\sim 0.05-0.35 \mathrm{Myr}$ and $\sim 0.45-0.75 \mathrm{Myr}, \varpi_{2}$ and $\varpi_{3}$ obviously have nearly the same precession rate as Jupiter. This complicates the secular evolution of asteroids in this planetary system, as we see below.

At high inclinations the Kozai resonance causes large coupled variations in the eccentricity and the inclination (Brasser et al. 2004), which finally results in instability. At low and moderate inclinations, the secular resonances $v_{3}$ and $v_{4}$ were found to affect the Earth Trojans ${ }^{6}$ (Morais 2001; Brasser \& Letho 2002). We note there are some disagreements in the locations of these secular resonances between different papers. We propose to present a detailed resonance map in a separate paper. Here we illustrate only the evolution of four typical orbits to show the effects of secular resonances that contribute to form the structures in the stability diagrams. Four orbits with a semimajor axis $a=0.9995 \mathrm{AU}$ and inclination $i=10^{\circ}, 22^{\circ}, 35^{\circ}, 42^{\circ}$ in Fig. 8 are selected. The evolutions of the resonant angles are presented in Figs. 14 and 15, respectively, and the eccentricities are presented in Fig. 16. The orbits with $i=10^{\circ}$ and $i=35^{\circ}$ are obviously in the stable windows, while the orbits with $i=22^{\circ}$ and $i=42^{\circ}$ are in the unstable gaps visible in Fig. 8.

Our preliminary calculations show that the most important secular resonances affecting the Earth Trojans are $v_{2}, v_{3}, v_{4}$, and $v_{5}$. According to the linear theory of secular perturbation, the average temporal variation in the eccentricity of a celestial body perturbed by a perturbing planet can be roughly approximated by

$\langle\dot{e}\rangle=C \sin \left(\Delta \varpi_{i}\right)=C \sin \left(\varpi-\varpi_{i}\right)$,

where $C$ is a negative constant determined by the mass of the planet and the Laplace coefficients (Murray \& Dermott 1999; Li et al. 2006), $\varpi$ and $\varpi_{i}$ are the perihelion longitudes of the celestial body and the planet, and $\Delta \varpi_{i}=\varpi-\varpi_{i}$ with $i=1, \ldots, 5$. If $0^{\circ}<\Delta \varpi_{i}<180^{\circ},\langle\dot{e}\rangle$ is less than zero and if $180^{\circ}<\Delta \varpi_{i}<$ $360^{\circ}$ then $\langle\dot{e}\rangle$ is greater than zero.

We note that for the orbit with $i=10^{\circ}$, from $t=0.05$ Myr to $0.3 \mathrm{Myr}$, the angles $\Delta \varpi_{2}, \Delta \varpi_{3}$, and $\Delta \varpi_{5}$ all librate around values

\footnotetext{
6 A Trojan precessing at the rate of the eigenfrequency $g_{3}$ (or $g_{4}$ ), which is the precession rate of the Earth (or Mars), is in the $v_{3}$ (or $v_{4}$ ) secular resonance.
}
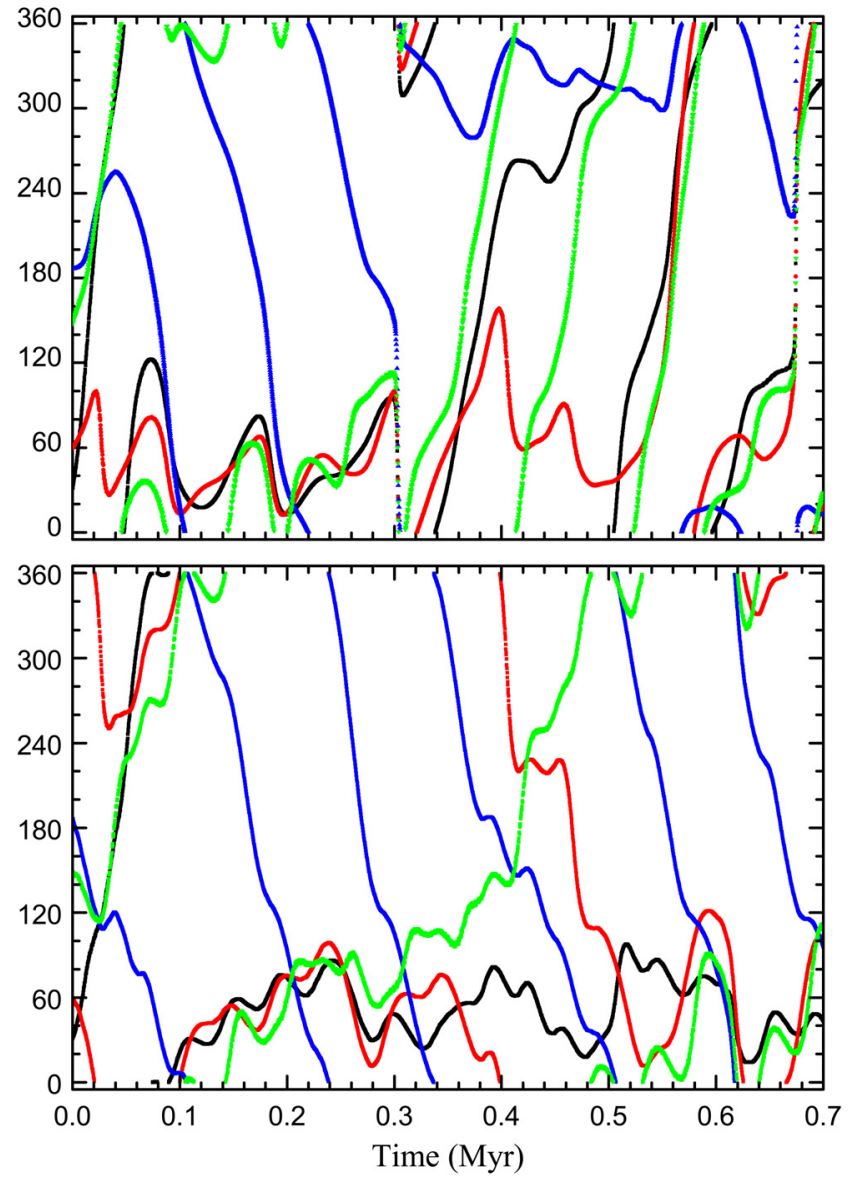

Fig. 14. The evolution of apsidal differences between the Trojans and the planets: $\Delta \varpi_{2}=\varpi-\varpi_{2}$ (black), $\Delta \varpi_{3}=\varpi-\varpi_{3}$ (red), $\Delta \varpi_{4}=\varpi-\varpi_{4}$ (blue), and $\Delta \varpi_{5}=\varpi-\varpi_{5}$ (green). The upper panel is for $i=10^{\circ}$ and the lower panel for $i=35^{\circ}$.

$\sim 40^{\circ}$ (see Fig. 14, upper panel). This libration affects (but not increases, according to Eq. (6)) the eccentricity of the Trojan. The overlap of these secular resonances introduces chaos into the motion, as can be seen in the irregular eccentricity evolution (black in Fig. 16). After $t=0.3 \mathrm{Myr}$, the $v_{2}$ and $v_{5}$ vanishes as the corresponding resonant angles circulate, but $\Delta \varpi_{4}$ begins to librate around a value larger than $180^{\circ}$, i.e. the $v_{4}$ resonance appears. Although this $v_{4}$ resonance may increase the eccentricity because $\Delta \varpi_{4}>180^{\circ}$, this eccentricity pumping effect is offset by the $v_{3}$ resonance, which is always present for $\Delta \varpi_{3}<180^{\circ}$.

Similar evolution occurs for $i=35^{\circ}$ (see Fig. 14, lower panel). However, in this case, before $t=0.1 \mathrm{Myr}, v_{3}$ and $v_{5}$ librate around high values, resulting in eccentricity excitation in this period (blue in Fig. 16). The $v_{4}$ resonance does not occur at all, while $v_{3}$ and $v_{5}$ are absent for about $0.1 \mathrm{Myr}$ from $0.4 \mathrm{Myr}$ to $0.5 \mathrm{Myr}$. Most of time, the resonant angles are smaller than $180^{\circ}$ and the eccentricity remains low.

The situation is quite different for $i=22^{\circ}$ (see Fig. 15, upper panel). The $v_{4}$ resonance exists with the resonant angle around $180^{\circ}$ until the Trojan is expelled from the 1:1 MMR at $t \sim 0.63 \mathrm{Myr}$. No other secular resonances are present until $t \sim 0.35 \mathrm{Myr}$, and the Trojan's eccentricity varies in correspondence with the circulation of the main secular angles $\Delta \varpi_{k}$ $(k=2,3,5)$. The most significant eccentricity excitation occurs when the $v_{3}$ resonance sets in at $t \sim 0.35 \mathrm{Myr}$. In this secular resonance, the eccentricity reaches 0.25 at $t=0.55 \mathrm{Myr}$ (red in 

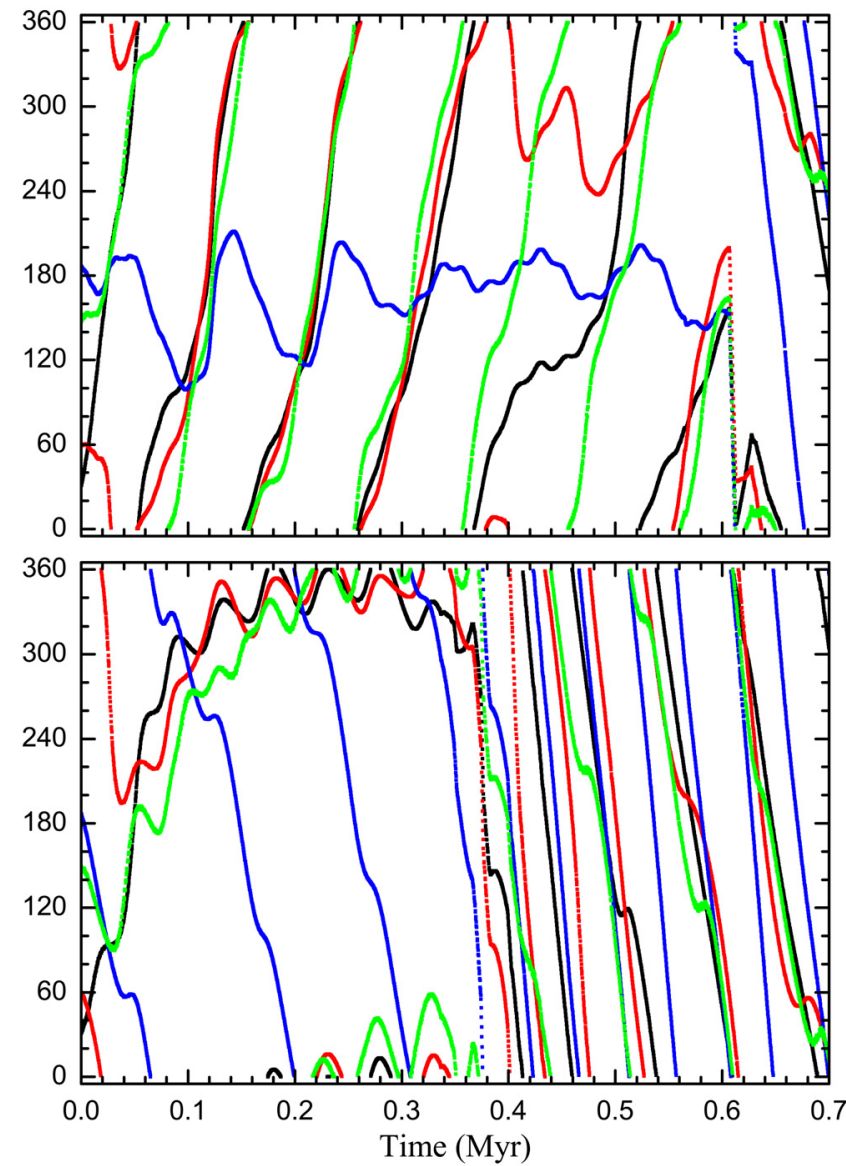

Fig. 15. The evolution of apsidal differences between the Trojans and the planets: $\Delta \varpi_{2}$ (black), $\Delta \varpi_{3}$ (red), $\Delta \varpi_{4}$ (blue), and $\Delta \varpi_{5}$ (green). The upper panel is for $i=22^{\circ}$ and the lower panel is for $i=42^{\circ}$.

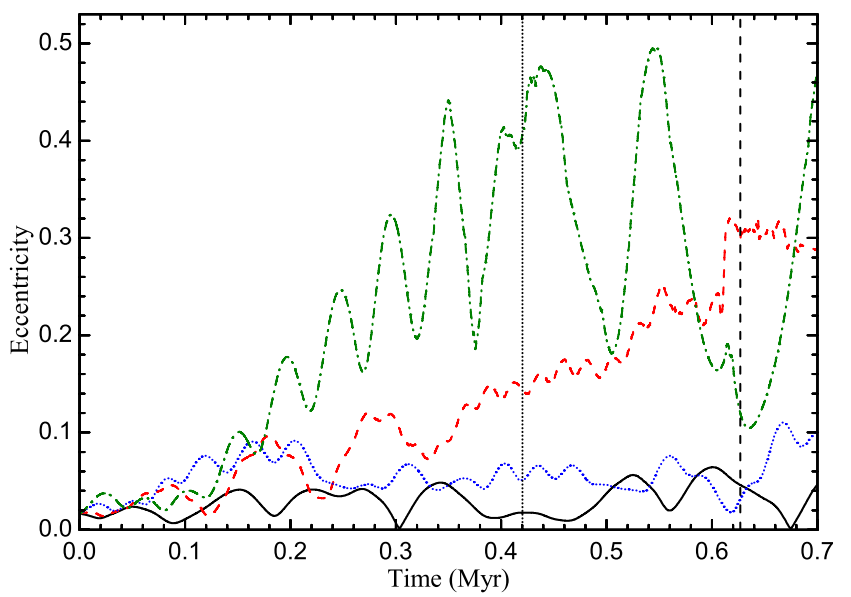

Fig. 16. The eccentricity evolution for four orbits. The solid curves represent the orbits with initial inclination $i=10^{\circ}$ (solid black), $22^{\circ}$ (dash red), $35^{\circ}$ (dot blue), and $42^{\circ}$ (dash-dot green), respectively. The vertical dash and dot lines mark the moments when the orbits escape from the 1:1 MMR for $i=22^{\circ}$ and $i=42^{\circ}$, respectively.

Fig. 16), where the Trojan may approach Venus closely, destabilizing the Trojan orbit.

For $i=42^{\circ}$ (see Fig. 15, lower panel), the resonant angles of $v_{2}, v_{3}$, and $v_{5}$ librate around high values, resulting in a quick excitation of the Trojan's eccentricity to 0.45 (red in Fig. 16).
Such a high eccentricity leads to its orbit crossing with Venus and Mars, and the Trojan finally becoming unstable.

On the basis of these results for typical orbital evolutions, we argue that the secular resonances $v_{2}, v_{3}, v_{4}$, and $v_{5}$ are all involved in determining the Trojans' stability. The resonances $v_{3}$ and $v_{5}$ play more important roles than the others.

\section{Conclusions}

We have investigated in full detail the stability of the recently found Earth Trojan asteroid $2010 \mathrm{TK}_{7}$ in different dynamical models. We derived a symplectic mapping based on the spatial elliptic restricted three-body problem to see the location of the mean orbit in the context of the phase space structure. We tested the validity of the simplified model with numerical integrations and found good agreement of the model on short timescales. We included the influence of the additional planets as well as the Moon to see their influences on the mean orbit of $2010 \mathrm{TK}_{7}$. We then performed a detailed numerical study to propagate the orbit of the Earth Trojan asteroid both forwards and backwards in time, together with 400 clone orbits to incorporate the possible errors in the orbital parameters induced by the observations. From our detailed study we were able to state the probability of both the capture and escape of an Earth Trojan and estimate the length of the time that the asteroid will stay close to the Lagrangian point $L_{4}$ of the Earth. To this end, we investigated in full detail not only the regime of parameters and initial conditions close to the asteroid actually found but instead on a grid of initial conditions and the parameter space $(a, i)$ using thousands of simulations, based on fictitious Earth Trojans. With these results we were able to define the region of stability and instability on both short and long timescales.

The main results of the present study can be summarized as follows: we can confirm the result of Connors et al. (2011) that the asteroid $2010 \mathrm{TK}_{7}$ lies in the tadpole regime of the Sun-Earth system. The orbital parameters indicate that the asteroid most probably became an $L_{4}$ Trojan some 1800 years ago and will either jump into the $L_{5}$ neighbourhood or enter a horseshoe orbit about 15000 years in the future. Before it moved into its current tadpole orbit, this asteroid was captured into the 1:1 MMR around 30000 years ago, and it may stay in the resonance for another 200000 years. The total lifetime of the asteroid (being in the 1:1 MMR) is less than 0.25 Myr. As a NEA, the closest approach of this asteroid to the Earth will be larger than 70 times the Earth-Moon distance when it is on a Trojan-like orbit. On short timescales, it is possible to predict the orbit in the spatial elliptic restricted three-body problem (Sun-Earth-asteroid) whereas on intermediate timescales the influence of the Moon has to be taken into account. In addition, on longer timescales the influence of the other planets in our Solar system cannot be neglected, since the asteroid is on a chaotic orbit with close encounters to the unstable equilibrium $L_{3}$. We expect the discovery of further interesting objects in the vicinity of the $L_{4}$ or $L_{5}$ equilibria of the Sun-Earth system. The long-term integrations show: for low inclinations, the stability region extends in terms of semi-major axis up to $\pm .01 \mathrm{AU}$. The size of it decreases with increasing inclination up to a threshold at about $i=20^{\circ}$. Another stability window opens up between $28^{\circ}$ and $40^{\circ}$ but disappears at larger angles. The preliminary results of our frequency analysis indicate that the $v_{2}, v_{3}, v_{4}$, and $v_{5}$ secular resonances are deeply involved in the motion of the Earth Trojans, and we have illustrated this with several examples.

From a qualitative point of view, our simulations have indicated that $2010 \mathrm{TK}_{7}$ is situated within an unstable region where 
all sorts of orbits such as horseshoes, tadpoles, and jumping ones are possible. We show - which is well confirmed - that the Earth Trojan moves on a temporary captured orbit. On the other hand, it is unclear why no other Trojans of the Earth have been found up to now because there are large stable regions for small inclinations, and for larger inclinations Trojans may also exist over very long timescales.

Acknowledgements. Z.L.Y. thanks the financial support by the National Natural Science Foundation of China (No. 10833001, 11073012, 11078001).

\section{References}

Bien, R., \& Schubart, J. 1984, Cel. Mech., 34, 425

Bowell, E., Holt, H. E., Levy, D. H., et al. 1990, BA\&AS, 22, 1357

Brasser, R., \& Letho, H. J. 2002, MNRAS, 334, 241

Brasser, R., Heggie, D., \& Mikkola, S. 2004, Celest. Mech. Dyn. Astron., 88, 123

Bretagnon, P. 1974, A\&A, 30, 141

Chambers, J. 1999, MNRAS, 304, 793

Connors, M., Wiegert, P., \& Veillet, C. 2011, Nature, 475, 481

Delva, M. 1984, Celest. Mech. Dyn. Astron., 34, 145

Dvorak, R., \& Schwarz, R. 2005, Celest. Mech. Dyn. Astron., 92, 19

Dvorak, R., Schwarz, R., Süli, Á., \& Kotoulas, T. 2007, MNRAS, 382, 1324

Dvorak, R., Bazsó, Á., \& Zhou, L. Y. 2010, Celest. Mech. Dyn. Astron., 107, 51

Eggl, S., \& Dvorak, R. 2010, Lect. Notes Phys, 790, 431

Érdi, B., Forgacs-Dajka, E., Nagy, I., \& Rajnai, R. 2009, Celest. Mech. Dyn. Astron., 104, 145

Ferraz-Mello, S. 1997, Celest. Mech. Dyn. Astron., 65, 421

Freistetter, F. 2006, A\&A, 453, 353

Hadjidemetriou, J. 1992, ZAMP, 37, 776
Hadjidemetriou, J. 1999, Celest. Mech. Dyn. Astron., 73, 65

Hadjidemetriou, J., \& Voyatzis, G. 2000, Celest. Mech. Dyn. Astron., 78, 137

Hanslmeier, A., \& Dvorak, R. 1984, A\&A, 132, 203

Laskar, J. 1999, Icarus, 88, 266

Lhotka, C. 2009, Celest. Mech. Dyn. Astron., 109, 175

Lhotka, C., Efthymiopoulos, C., \& Dvorak, R. 2008, MNRAS, 384, 1165

Li, J., Zhou, L.-Y., \& Sun Y.-S. 2006, Chin. J. Astron. Astrophys., 6, 588

Lichtenegger, H. 1984, Celest. Mech. Dyn. Astron., 34, 357

Mainzer, A., Bauer, J., Grav, T., et al. ApJ, 731, 53

Michtchenko, T. A., \& Ferraz-Mello, S. 2002, AJ, 122, 474

Mikkola, S., \& Innanen, K. 1992, AJ, 104, 1641

Morais, M. H. M. 2001, A\&A, 369, 677

Morais, M. H. M., \& Morbidelli, A. 2002, Icarus, 160, 1

Morbidelli, A., Levison, H. F., Tsiganis, K., \& Gomes, R. 2005, Nature, 435, 462

Murray, C. D., \& Dermott, S. F. 1999, Solar System Dynamics (Cambridge: Cambridge Univ. Press)

Nesvorný, D., \& Dones, L. 2002, Icarus, 160, 271

Nesvorný, D., \& Vokrouhlický, D. 2009, AJ, 137, 5003

Nesvorný, D., Thomas, F., Ferraz-Mello, S., \& Morbidelli, A. 2002, Celest. Mech. Dyn. Astron., 82, 323

Rabe, E. 1967, AJ, 72, 10

Robutel, P., \& Gabern, F. 2006, MNRAS, 372, 1463

Robutel, P., Gabern, F., \& Jorba, A. 2005, Celest. Mech. Dyn. Astron., 92, 53

Scholl, H., \& Marzari, F. 2004, Lun. Plan. Sci. Conf., 35, 1107

Scholl, H., Marzari, F., \& Tricario, P. 2005, AJ, 105, 130

Schwarz, R., \& Dvorak, R. 2011, Celest. Mech. Dyn. Astron., submitted

Schwarz, R., Gyergyovits, M., \& Dvorak, R. 2004, Celest. Mech. Dyn. Astron., 90,139

Tabachnik, S. A., \& Evans, N. W. 2000, MNRAS, 319, 63

Tsiganis, K., Dvorak, R., \& Pilat-Lohinger, E. 2000, A\&A, 354, 1091

Wiegert, P. A., Innanen, K. A., \& Mikkola, S. 1997, Nature, 387, 685

Zhang, S.-P., \& Innanen, K. A. 1995, AJ, 96, 1995

Zhou, L.-Y., Dvorak, R., \& Sun, Y. S. 2009, MNRAS, 398, 1217

Zhou, L.-Y., Dvorak, R., \& Sun, Y. S. 2011, MNRAS, 410, 1849 